\title{
Estimating the impact of variable renewable energy on base-load cycling in the GB power system
}

\author{
Patrick de Mars*, Aidan O’Sullivan, Ilkka Keppo \\ UCL Energy Institute, University College London, Central House, 14 Upper Woburn Place, London, WC1H ONN, UK
}

\section{A R T I C L E I N F O}

\section{Article history:}

Received 9 October 2019

Received in revised form

16 December 2019

Accepted 25 January 2020

Available online 30 January 2020

\section{Keywords:}

Power plant cycling

Renewable energy

Data analysis

Regression

\begin{abstract}
A B S T R A C T
Between 2009 and 2017 the share of wind and solar energy sources in the GB electricity generation mix increased from $2.5 \%$ to $17 \%$. Due to the variable nature of these renewable sources, large thermal power stations designed for constant base-load operation have been required to operate more flexibly to compensate for fluctuations in renewable generation. This flexible operation results in increased thermal stress and reduced efficiency causing increased operation, maintenance and fuel costs for these assets. In this paper we present the results of what is, to the best of our knowledge, the first empirical study on the impact of renewables generation on startups, ramping and part-loading (collectively, 'cycling') of baseload generators. We develop regression models using half-hourly generation data from 2009 to 2017 that capture the impact of increased renewable penetration while taking into account confounding factors including seasonality and demand. We find that with 2009-levels of renewable generation, cycling in 2017 would have been less severe, with $20 \%$ fewer startups. We also present estimates for cycling under National Grid Future Energy Scenarios to 2030 with implications for investment in generation assets. Additionally, the dataset derived in this research is made available and comprises the first open-access dataset on cycling.
\end{abstract}

(c) 2020 Published by Elsevier Ltd.

\section{Introduction}

Falling capital costs of renewable generation technologies, as well as greater acceptance of the threat of climate change, are leading to an increase in the penetration of variable renewable energy (VRE) in power systems globally [1]. However, power systems are comprised of complex interdependencies and VRE has consequences for existing conventional power plants within the system. VRE operates at very low marginal cost, displacing conventional power stations in the generation mix when available. Except by curtailment, VRE cannot be easily controlled, and there is a requirement for the conventional generation mix to provide the flexibility required to compensate for the added uncertainty and variability, ensuring the balance of supply and demand [2].

There is a large body of energy systems modelling research investigating power systems under high levels of VRE penetration: for a thorough review, see [3]. However, there is a shortage of datadriven research into the impacts of VRE on power systems. Top-

\footnotetext{
* Corresponding author.

E-mail address: patrick.demars.14@ucl.ac.uk (P. de Mars).
}

down empirical research of this kind benefits in that it does not rely on high-resolution simulation of power systems. In contrast, bottom-up simulation studies require accurate consideration of factors such as transmission and generator constraints and complex power markets to accurately simulate cycling [4,5], which is often challenging due to computational expense or data unavailability. As we will show in Section 2, the study of the GB power system (England, Scotland and Wales) from 2012 to 2020 [6] did not predict the increases in cycling we observe in the historical data by 2017. This provides motivation for adopting an empirical approach to studying the impact of increased VRE penetration on cycling.

In this paper, we present analysis of a novel dataset containing 9 years of half-hourly operational data from the GB power system, examining the relationship between increased VRE penetration, which increased from 2.5 to $17 \%$ during the period (Fig. 1), and operational behaviour of conventional generation. In particular we focus on cycling, which we investigate by considering the number of startups, severe ramping events and average load factor (measuring the extent of part-loading). In addition, we calculate annual capacity factors for each generator.

Estimating the extent of cycling is important for estimating operating costs of power plants due to the increased O\&M costs 


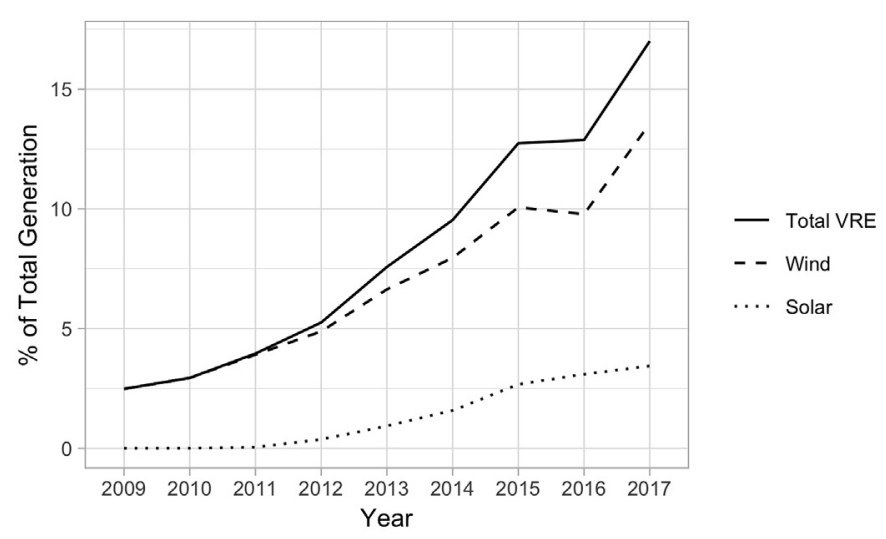

Fig. 1. Annual VRE penetration 2009-2017, rising from 2.5\% to $17 \%$. VRE penetration is calculated using total VRE generation including estimations for unmetered sources.

[7-9]. Startups frequently cost of the order $£ 10,000$ for CCGTs ${ }^{1}$ while part-loading and ramping also incur increased fuel costs (due to reduced efficiency) and increased long-term O\&M costs (due to thermal stress), respectively.

The behaviour of power systems at a weekly resolution is highly variable due to seasonal changes, developments in demand and changes in the generation mix. In order to understand the relationship between cycling and VRE penetration at this resolution, we need to take into account these additional factors. This requires the development of models which we present in this paper that capture and quantify the impact of VRE on the power system. We use regression analysis for better interpretability as opposed to methods which might provide stronger predictive power. These models can be incorporated into existing scenario modelling tools for the GB energy system to more accurately capture the impact of VRE on cycling. While several studies have investigated VRE and cycling in simulation [4,5,9-11], to the best of our knowledge this is the first to use empirical methods.

The main contributions of this work are as follows. First, we present regression models which describe the impact of variables in the power system on cycling and which can be used as module for scenario modelling. We also estimate that the number of startups per week would have been $20 \%$ lower, load factor $10 \%$ higher and severe ramping events per week 6\% lower in 2017 had VRE generation remained at 2009 levels. Second, we use our models to estimate cycling in National Grid Future Energy Scenarios up to 2030, beyond the most recent estimates to 2020 [6]. Our predictions suggest that cycling would become considerably more severe under all scenarios, with startups per generator increasing by up to $150 \%$, ramping events per generator by up to $190 \%$, and average load factor decreasing by up to $8 \%$. As a final contribution, the dataset developed in this research is made available to the wider research community and is the first of its kind.

The paper is organised as follows. In the next section, we review current research into the impact of VRE on power systems. We then present some exploratory analysis of the data which motivates the following section outlining our methodology. The fitted regression models capturing the relationship between the cycling variables and VRE generation are presented in Section 5.1 and applied to Future Energy Scenarios in Section 5.2. We discuss our findings in Section 6 before making some concluding remarks.

\footnotetext{
${ }^{1}$ Estimates from [8]. Median lower bound warm start costs for CCGT plants are reported as $\$ 55$ per MW capacity.
}

\section{Literature review}

In this section we provide an overview of simulation studies which have investigated cycling in high-VRE systems. We also present literature regarding the cycling costs resulting from increased thermal stress on power stations.

The impact of VRE penetration on power systems has been assessed from several perspectives including frequency control [12-15], increased reserve requirements [16-18], transmission network reinforcement $[19,20]$ and voltage stability [21,22]. Most relevant to our research are those studies which investigate the operation of conventional power stations under VRE penetration, which has generally been assessed by grid simulation using dispatch models [2,4,5,9-11,23-29]. Simulation studies on cycling have been conducted on a range of power systems including the U.S. Western Interconnection [27], European interconnections [4,28], ERCOT [11], Ireland [10,11,23], GB [2], the Netherlands [24], Germany [5,9,25], Finland [11,30], Spain [29] and California [26]. The GB power system has been studied in simulation in [6], which makes predictions for ramping, part-loading and startups to 2020 under four scenarios. To the best of our knowledge, no empirical studies of base-load cycling under VRE penetration exist in the literature.

The study of the GB power system between 2012 and 2020, with VRE penetration rising from $5.6 \%$ to between 17 and $21 \%$ is particularly relevant to this research [6]. Startups per generator are predicted to change by between $\pm 10 \%$ under different scenarios between 2012 and 2020 . Changes of approximately $\pm 20 \%$ are predicted for ramping (measured by rate of change in production) and the proportion of time spent at minimum stable levels (representing part-loading) for gas power stations is predicted to remain roughly stable. Direct comparison with our analysis of the historical data in Section 3.2 is difficult due to the different metrics used. However, we will show that cycling increased on a per-generator level between 2012 and 2017: startups increased by 17\%, ramping events by $39 \%$ and load factor decreased by around $1 \%$.

Cycling operation of power stations is often quantified by the frequency of startups (when a power station begins generating), average load factor (the instantaneous output of a power station divided by its capacity during online hours; thus measuring partloading) and ramping requirements (load adjustments required to meet fluctuations in demand) [10]. Several simulation studies have considered penetrations up to 40\% VRE penetration on different power systems (Germany [9], Ireland [10], Finland, Ireland and Texas [11]). They find the total number of startups increases by 3-6 times compared with a scenario with no VRE. For load factor, [10] reports a decrease of $6 \%$ for coal power stations while [11] finds a decrease of $10-15 \%$ over all base-load generators. Ramping, measured by rate of change in production, is found to increase by around $50 \%$ in [9], while in [10] the number of hours of severe ramping (defined as a change in load of over $50 \%$ in under $1 \mathrm{~h}$ ) per year increases sharply from $<10$ to approximately 30 per CCGT between $30 \%$ and $40 \%$ VRE penetration. Other simulation studies at different penetrations predict total startups to increase: by roughly double from $10 \%$ to $40 \%$ penetration for a test system based on the Spanish grid [29] and by approximately 6 times from $0 \%$ to $27 \%$ for Ireland [23].

Transmission constraints have a substantial impact on cycling operation [4] but may be difficult to include in dispatch models due to computational budget constraints. More conservative increases are predicted by those studies which include network constraints at high resolution as well as developments in the generation mix in response to rising VRE penetration [4,5]. Startups increase by 4-23\% between 2013 and 2020 in simulations of the Central and Western European interconnection in [4], and by $81 \%$ between 
2013 and 2030 in Germany [5]. Few simulations studies have modelled the transmission network at high resolution due to the data and computational budget requirements. In addition, other factors such as multiple complex power markets are not typically included in simulation studies, but may impact dispatch decisions and levels of base-load cycling. There is therefore a demand for greater empirical evidence on the impacts of VRE on power systems without these reliances.

Costs from cycling derive from thermal stresses causing increased operation and maintenance $(\mathrm{O} \& \mathrm{M})$ costs and higher rates of forced outage $[8,9,31]$. Starting up a power station, which can cause severe stress on physical components, reduces the lifetime of the plant and results in increased O\&M costs over the power station's lifetime. In addition, rated efficiency decreases with load factor (by around $10-15 \%$ from 100 to $40 \%$ load for CCGTs [9,32]) and frequent startups causing increased fuel costs. Overall, the indirect costs of a CCGT startup outweigh the direct costs of fuel, emissions and auxiliary services by 8 times [9]. Accounting for indirect costs, the cost for starting up a CCGT power station are estimated between \$32-93 per MW capacity in [8]. Due to the large time delays between cycling and incurred costs resulting from O\&M and forced outages, cycling costs are difficult to measure and often underestimated [33].

The large number of simulation studies show broad consensus that VRE penetration causes increased cycling of base-load power stations. It is also known that increased cycling results in significant increases in total operating costs of power stations. However, there is considerable uncertainty regarding the magnitude of the impact of VRE on cycling, which is dependent on the power system under consideration. This motivates our study which aims to quantify the impact of VRE generation on cycling in the GB power system using an empirical approach. In addition, developing a model for cycling under VRE penetration would provide a novel alternative to simulation approaches for estimating cycling in future GB power systems. While cycling in the GB power system has been forecast to 2020 using a simulation method in [6], the literature lacks up-todate estimates for the next decade under different scenarios. The following section outlines the cycling data derived for this research and some exploratory analysis.

\section{Data \& exploratory analysis}

Here we describe the primary data used in this research and definitions of the derived cycling variables. We briefly present analysis of the cycling data from 2009 to 2017.

\subsection{Data}

Cycling data was derived for the period 2009-2017 using data from the Elexon P114 dataset [34] and the National Grid demand data [35]. The derived dataset has been made available under an open data license. ${ }^{2}$ The Elexon data gives half-hourly metered generation data for all generators connected at the transmission level as well as some embedded generators such as wind farms and open-cycle gas turbine (OCGT) plants. 91 wind farms, 70 gas-fired and 17 coal-fired power stations are recorded in the data. For power stations with several generating modules, the data records readings from as many meters: 132 meters from wind farms are recorded in the data, 122 from gas-fired power stations, and 63 from coal-fired stations. In this research, meter readings were not aggregated by power station, and henceforth references to generators and power stations correspond to individual meters.

\footnotetext{
${ }^{2}$ Cycling data is available at: https://doi.org/10.5281/zenodo.3474820.
}

Fuel type was determined primarily using Elexon's BM Reports [36]. There was a small number of unmatched cases which we determined on an individual basis using a range of online sources. Due to the considerable developments in the UK electricity generation mix, several power stations were either commissioned or decommissioned during the period and are not active throughout the data. When they are active, these power stations were included in the calculation of cycling variables.

Base-load power stations were defined as all gas- and coal-fired power stations with capacities of at least 100 MW. Capacity was inferred from the highest observed output in a single settlement period, as this could not be obtained from existing data sources for all generators. Verifying with known capacities, this was found to be an accurate estimate for nameplate capacity.

Using the Elexon data, we calculated total startups, total severe ramping events and average load factor at the daily resolution. Startups were calculated by counting generator production changing from $<1 \mathrm{MWh}$ to $\geq 1 \mathrm{MWh}$ (to protect against metering artefacts) in consecutive settlement periods. We defined severe ramping events as a change in production of $\geq 25 \%$ of a generator's maximum output between consecutive settlement periods. Load factor was defined as the average instantaneous output of a generator divided by its production during online periods only. Daily average load factor over the entire base-load generation mix was then calculated by taking the mean over all generators. Lastly, we calculated weekly aggregations of these variables to smooth variation caused by weather, major events and other random effects. All results presented in this paper are for the weekly aggregations. The variables at the daily resolution are shared in the open dataset.

We also calculated annual capacity factor for each generator. Capacity factor was defined as in [10]: the ratio of observed generation to maximum possible generation over a given period, assuming complete plant availability with no scheduled maintenance. While load factor represents the extent of part-loading, capacity factor represents overall generator utilisation.

The number of active base-load generators each year was calculated as the count of base-load generators (gas and coal-fired power stations with capacity $\geq 100 \mathrm{MW}$ ) with at least one startup.

Total VRE generation was calculated as the sum of unmetered wind generation, solar generation (estimates from National Grid demand data) and metered wind generation (from the Elexon data). Total generation was calculated as the sum of all metered generation (from the Elexon data) with interconnection imports, unmetered wind generation and solar generation. VRE penetration is the ratio of VRE generation to total generation.

\subsection{Historical cycling trends}

Total weekly startups rose from 160 to 201 between 2009 and 2012, then remained stable until 2015, before decreasing to 173 in 2017. The distribution of cold, warm and hot starts (based on the length of inactivity prior to startup) remained roughly stable apart from a small relative increase in cold and hot starts during 2012-2015. The number of weekly startups is highly seasonal, peaking in winter and dropping considerably in summer.

The average number of severe ramping events per week fluctuated between a minimum of 451 in 2009 and a maximum of 532 in 2016. Like startups, severe ramping events show strong seasonal trends, being highest in winter and lowest in summer. Average load factor of base-load generators decreased steadily over the period, from $77 \%$ in 2009 to $71 \%$ in 2017 . VRE penetration is weakly correlated with startups (0.011) and severe ramping events (0.10). This suggests that links between VRE penetration and startups and ramping are likely to be masked by other exogenous factors such as 


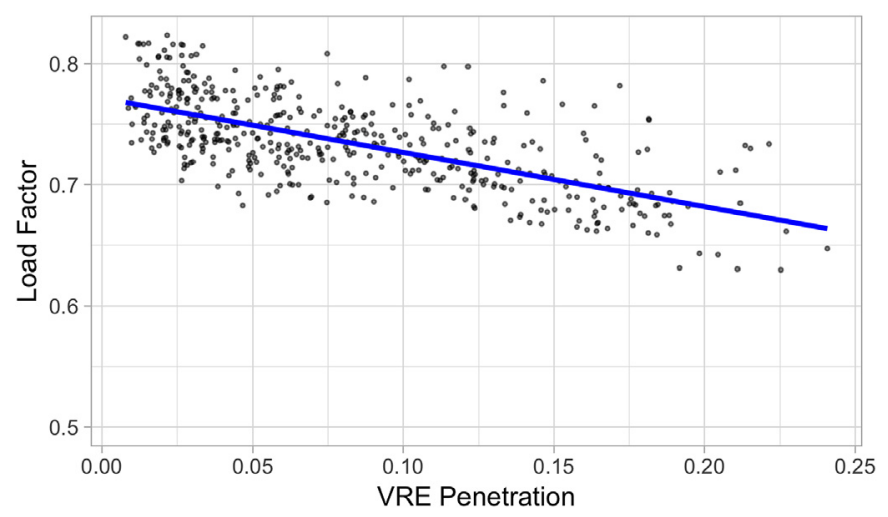

Fig. 2. Observed average weekly load factor of base-load generators against VRE penetration. Load factor is negatively correlated $(-0.66)$ with VRE penetration.

demand. VRE penetration is more strongly correlated with load factor $(-0.66)$, as shown in Fig. 2.

The number of active base-load generators was highest in 2012 at 115 , then decreased steadily to 85 in 2017 . This has resulted in increasing numbers of startups and ramping events per generator, which were respectively 31\% and 38\% higher in 2017 than in 2009.

\subsection{Distribution of cycling variables}

The distributions of annual aggregations of the cycling variables at the generator level in 2009 and 2017 are shown in Fig. 3. In all four cases, the distributions show shifts towards stronger cycling of base-load generators: in general, generators operated at lower capacity and load factors and experienced more frequent ramping events and startups.

We used hypothesis tests to determine whether the apparent increase in cycling severity was statistically significant. Kolmogorov-Smirnov hypothesis tests on these distributions show statistically significant differences (with a 5\% significance threshold) in the distributions for all four variables between 2009 and 2017. By comparison, running the same tests for 2009 and 2010, we could not reject null hypotheses for any of the cycling variables. We therefore conclude that the data shows a statistically significant worsening of cycling between 2009 and 2017.

\section{Methodology}

Having presented some initial observations of the cycling data, we now describe our methodology for developing the cycling regression models. As shown in Section 3, weak correlation between variables did not immediately reveal links between VRE penetration and cycling, suggesting that other factors such as demand, changes to the generation mix and seasonality effects needed to be accounted for using a multivariate model. As the aims of this research were both to quantify the impact of VRE on cycling and to provide a model for predicting startups in future power systems, we chose to use multivariate regression as opposed to alternative methods which may have produced better predictive power but would lack interpretive value (such as neural networks). We used combined VRE generation (wind plus solar generation) in the models as annual solar penetration was <1\% until 2014. 459 complete weeks were used to fit the models. We compared models partly using mean absolute percentage error (MAPE) and rootmean-square error (RMSE) of predictions for 2017 when trained on data from 2009 to 2016. The models reported in Section 5 are trained on the entire period 2009-2017 inclusive. As count data, startups and severe ramping events were Poisson-distributed and we used Poisson regression for these models. For load factor, we used ordinary least squares.

The regression model for the number of weekly startups $s$ was defined as follows:

$$
\ln s=\beta_{0}+\beta_{1} x+\beta_{2} g+\beta_{3} g^{2}+\beta_{4} n+\beta_{5} \sin (2 \pi \theta)+\beta_{6} \cos (2 \pi \theta)
$$

where $x$ is total VRE generation (GWh), $g$ is the total generation from all fuel types (GWh), $n$ is the number of base-load generators, and $\theta$ is the year fraction (week index as proportion of the number of complete weeks in the year). $\beta_{i}$ are regression coefficients. The sine and cosine transformations aim to capture the annual cycle observed in startups. A quadratic relationship was observed between startups and total generation, so a second-order term was included for this variable.

For load factor $l$, the regression model was defined as:

$$
l=\beta_{0}+\beta_{1} x+\beta_{2} g+\beta_{3} n
$$

For load factor, seasonality trends were found to be welldescribed by annual variation in total generation, so we did not include the year fraction variables. In addition, it was more appropriate to model a linear relationship between load factor and total generation.

The model for the number of severe ramping events $r$ was defined as:

$\ln r=\beta_{0}+\beta_{1} x+\beta_{2} g+\beta_{3} g^{2}+\beta_{4} n+\beta_{5} \sin (2 \pi \theta)+\beta_{6} \cos (2 \pi \theta)$

Like for startups, a quadratic relationship was observed between ramping events and total generation, and seasonality effects were not captured by variations in demand alone.

\section{Results}

In this section we present the results of our model estimates and forecast cycling under Future Energy Scenarios.

\subsection{Regression models}

Having found significant developments in the cycling variables and described our methodology, we now present the results of model estimation using maximum likelihood for startups, load factor and severe ramping events and discuss model performance. The models are validated by training on data up to and inclusive of 2016 and testing on 2017 data. The full models are then reported and we provide two practical interpretations of the coefficients. First, we report the change in the response variable resulting from an increase of $50.4 \mathrm{GWh}$ in total weekly VRE generation (chosen as the total output of a $1 \mathrm{GW}$ generator operating at $30 \%$ capacity factor). Second, we report estimates for the response variables in 2017, had VRE generation remained at 2009 levels, with all other variables set to 2017 levels (annual averages).

\subsubsection{Startups}

Observed weekly base-load startups for 2017 and those predicted using the regression model trained on data from 2009 to 2016. Our predictions closely follow the seasonal cycle and match the data with a MAPE of $9.7 \%$.

Observed weekly base-load severe ramping events for 2017 and those predicted using the regression model trained on data from 2009 to 2016. The model achieves a MAPE of $10.2 \%$.

The regression model for weekly startups trained on all data (2009-2017) is presented in Table 1. When trained on data from 

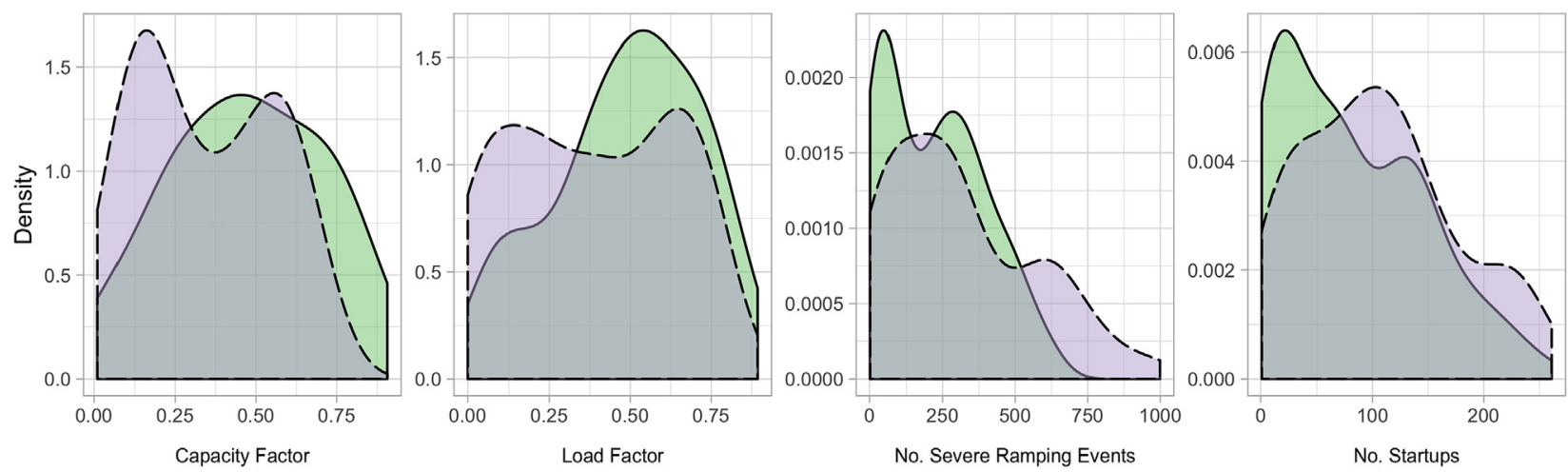

Year

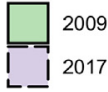

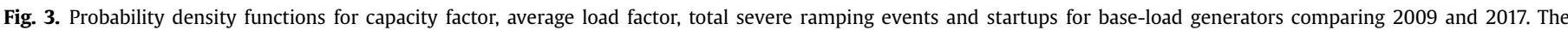
distributions show shifts towards more severe cycling for all 4 variables.

Table 1

Poisson regression model for weekly startups of base-load generators.

\begin{tabular}{|c|c|c|c|c|}
\hline Term & Estimate & Confidence: $5 \%$ & Confidence: 95\% & p-value \\
\hline Intercept & -2.53 & -3.31 & -1.75 & $\approx 0$ \\
\hline Total VRE generation (GWh) & $2.80 \mathrm{e}-04$ & $2.44 \mathrm{e}-04$ & $3.16 e-04$ & $\approx 0$ \\
\hline Total generation (GWh) & $1.99 e-03$ & $1.76 \mathrm{e}-03$ & $2.23 e-03$ & $\approx 0$ \\
\hline Total generation $(\mathrm{GWh})^{2}$ & $-1.47 e-07$ & $-1.65-07$ & $-1.29 \mathrm{e}-07$ & $\approx 0$ \\
\hline No. base-load generators & $9.23 e-03$ & $8.19 \mathrm{e}-03$ & $10.3 e-03$ & $\approx 0$ \\
\hline $\sin ($ year fraction) & $-11.0 \mathrm{e}-02$ & $-12.3 e-02$ & $-9.71 \mathrm{e}-02$ & $\approx 0$ \\
\hline $\cos$ (year fraction) & $7.80 \mathrm{e}-02$ & $5.53 e-02$ & $10.1 \mathrm{e}-02$ & $\approx 0$ \\
\hline
\end{tabular}

2009 to 2016, the model achieves a RMSE of 19.6 startups and a MAPE of 9.7\% when predicting startups in 2017. The predicted and actual weekly startups are plotted in Fig. 4. With 50.4 GWh of VRE generation the model predicts a resulting increase in total startups of $1.4 \%$. If VRE generation had not increased between 2009 and 2017, startups would have fallen to 138 per week in 2017, compared with 173 observed in the data. The number of startups is lowest in May and highest in November.

\subsubsection{Load factor}

We regress load factor against VRE generation, total generation and number of generators. Though there is a clear seasonal cycle in load factor (highest in Winter, lowest in Summer), this can be captured by similar variation in total generation and the seasonal variables do not improve the model. The full regression model is presented in Table 2 and has an adjusted R-squared of 0.69 . Trained

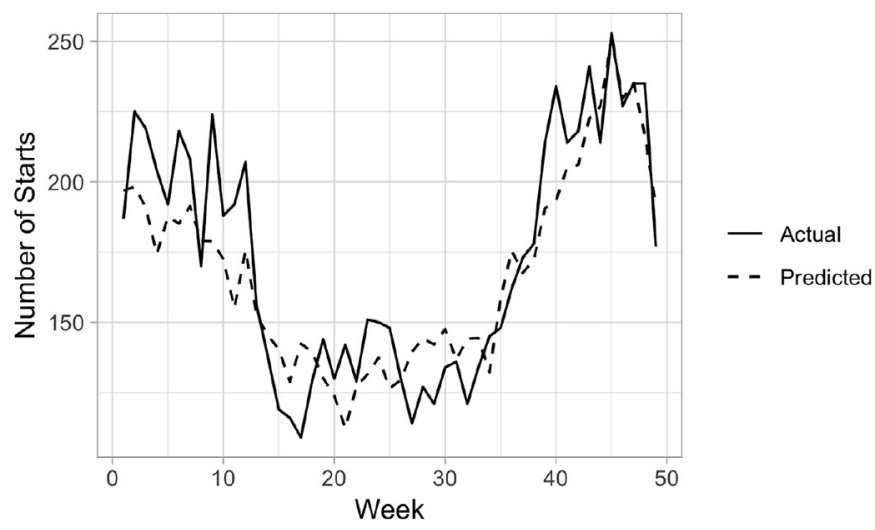

Fig. 4. Observed weekly base-load startups for 2017 and those predicted using the regression model trained on data from $2009\{2016$. Our predictions closely follow the seasonal cycle and match the data with a MAPE of $9.7 \%$. on data from 2009 to 2016 and testing on 2017, the model achieves a RMSE of 0.038 and MAPE of $4.3 \%$. Load factor increases with total generation, although the magnitude of this effect is around a third that of VRE generation. Load factor also decreases by $0.13 \%$ for each additional active base-load generator. An additional $50.4 \mathrm{GWh}$ of VRE generation results in a decrease of $0.46 \%$ in average load factor. With 2009 levels of VRE generation, load factor would have remained stable at $78 \%$ in 2017 , compared with the observed decrease to $71 \%$.

\subsubsection{Severe ramping events}

The regression model for severe ramping events is presented in Table 3. Trained on data from 2009 to 2016 and testing on 2017, this model achieves a RMSE of 55.9 with a MAPE of 10.2\% (Fig. 5). Our model predicts severe ramping events to increase by $0.36 \%$ with an additional 50.4 GWh VRE generation per week. At 2009 levels of VRE generation, the number of weekly severe ramping events would have been 484 , compared with 516 observed in the data. The number of severe ramping events is lowest in June and highest in December.

\subsection{Future Energy Scenarios}

We used the fitted regression models to predict average load factor, total weekly startups and severe ramping events from 2018 to 2030 under National Grid's 2017 Future Energy Scenarios (FES) [37], which give projections for generation by fuel type. We use the regression models to estimate cycling under each scenario, all other factors being equal. Increases in factors including storage, demandside response and interconnection are likely to impact the actual development of cycling but cannot be considered using the regression models.

VRE penetration and weekly generation in 2030 are highest in the Two Degrees scenario at $40 \%$ and 6.6 TWh. Steady State has the lowest VRE penetration and total generation (30\% and 5.9 TWh). 
Table 2

Linear regression model for average weekly load factor of base-load generators.

\begin{tabular}{|c|c|c|c|c|}
\hline Term & Estimate & Confidence: $5 \%$ & Confidence: $95 \%$ & p-value \\
\hline Intercept & 0.699 & 0.664 & 0.735 & $\approx 0$ \\
\hline Total VRE generation (GWh) & $-9.15 e-05$ & $-10.1 \mathrm{e}-05$ & $-8.21 \mathrm{e}-05$ & $\approx 0$ \\
\hline Total generation $(\mathrm{GWh})$ & $3.39 e-05$ & $3.09 e-05$ & $3.69 e-05$ & $\approx 0$ \\
\hline No. base-load generators & $-1.25 \mathrm{e}-03$ & $-1.54 \mathrm{e}-03$ & $0.955 \mathrm{e}-05$ & $\approx 0$ \\
\hline
\end{tabular}

Table 3

Poisson regression model for weekly severe ramping events of base-load generators.

\begin{tabular}{|c|c|c|c|c|}
\hline Term & Estimate & Confidence: $5 \%$ & Confidence: 95\% & p-value \\
\hline Intercept & 1.47 & 1.00 & 1.93 & $\approx 0$ \\
\hline Total VRE generation (GWh) & $7.14 \mathrm{e}-05$ & $4.99 \mathrm{e}-05$ & $9.28 \mathrm{e}-05$ & $\approx 0$ \\
\hline Total generation (GWh) & $1.42 \mathrm{e}-03$ & $1.28 \mathrm{e}-03$ & $1.56 \mathrm{e}-03$ & $\approx 0$ \\
\hline Total generation $(\mathrm{GWh})^{2}$ & $-1.02 \mathrm{e}-07$ & $-1.12 \mathrm{e}-07$ & $-0.915 e-07$ & $\approx 0$ \\
\hline No. base-load generators & $-1.55 \mathrm{e}-03$ & $-2.17 e-03$ & $-0.917 \mathrm{e}-03$ & $\approx 0$ \\
\hline $\sin ($ year fraction) & $-6.21 \mathrm{e}-02$ & $-6.99 e-02$ & $-5.44 \mathrm{e}-02$ & $\approx 0$ \\
\hline $\cos$ (year fraction) & 0.145 & 0.131 & 0.158 & $\approx 0$ \\
\hline
\end{tabular}

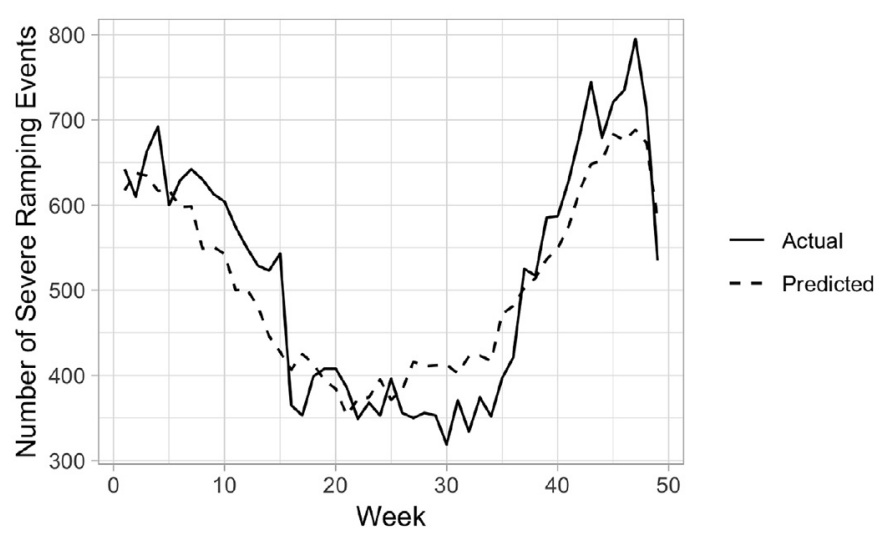

Fig. 5. Observed weekly base-load severe ramping events for 2017 and those predicted using the regres-sion model trained on data from 2009\{2016. The model achieves a MAPE of $10.2 \%$.

The number of base-load generators in operation was determined using predicted base-load capacity from the FES, using an average base-load generator capacity of 455 MW (matching FES capacities with the number of active generators in the Elexon data for 2016). The number of baseload generators in 2030 range from 39 (Slow Progress) to 80 (Steady State). Since the FES data predicts actual generation before losses, we scaled down the FES predictions by $7 \%$ (based on a comparison with the Elexon data for 2016).

The predictions for all cycling variables including 95\% confidence intervals are presented in Fig. 6. The largest increases in startups are under the Steady State (low prosperity, low green ambition) and Two Degrees (high prosperity, high green ambition) scenarios, where startups are predicted to rise to up to 209 and 206 per week, respectively. All scenarios also see a decrease in startups until around 2022 as demand and base-load capacity decrease, continuing the trend seen between 2015 and 2017. The lowest increase in startups is for Slow Progress (low prosperity, high green ambition), where we predict 176 startups per week in 2030. Compared with a baseline of 173 startups per week (mean for 2017), this represents increases of $1.6-21 \%$. Average load factor remains stable until 2022, before decreasing at a similar rate in all scenarios to $63-65 \%$. The number of severe ramping events rises consistently in all scenarios, by between $6.9 \%$ (Steady State) and 35\% (Two Degrees).
Fig. 7 shows the development in per-generator startups and severe ramping events, where the number of active generators is determined using projected base-load capacities from the FES. While total startups increase by up to $21 \%$, there is a much larger increase in startups per generator of between 28 (Steady State) to $150 \%$ (Two Degrees) due to declining numbers of base load generators. Similarly, severe ramping events per generator increase by between 14 and 190\%.

Predicted average load factor, total severe ramping events and total startups per week under Future Energy Scenarios with 95\% confidence bands. Load factor decreases similarly under all scenarios. The number of severe ramping events increases most severely under the Two Degrees scenario of high VRE penetration and high demand. Startups decrease in all scenarios before increasing above 2017 levels. Startups are high in Steady State in 2030 due to a large base-load mix, and in Two Degrees due to high demand and VRE penetration.

Predicted severe ramping events and startups per generator per week under Future Energy Scenarios with 95\% confidence bands. Two Degrees and Slow Progress are the highest cycling scenarios for both per-generator ramping and per-generator startups, as these scenarios have the smallest base-load mix. Per-generator increases under the Steady State scenario are comparatively slow, due to large predicted increases in CCGT capacity.

\section{Discussion}

Our initial analysis of the data showed that effects of VRE penetration on cycling were often obscured by other factors. Section 3.2 showed that total startups remained largely stable between 2009 and 2017, despite rising VRE penetration. The regression model in Table 1 explains that total startups were prevented from increasing due to decreasing demand and fewer base-load generators in operation (17\% fewer in 2017 than 2009) over the period, which effectively cancelled out the impact of increased renewables. In addition, heavy cycling of new-build OCGTs after 2015 is likely to have helped prevent rises in base-load startups. While total cycling requirements did not change considerably, weekly startups and severe ramping events per active generator were found to have increased by 31\% and 38\% respectively between 2009 and 2017, exceeding predictions in [6] and indicating the displacement of individual generators into cycling operation. Combined with the observed decrease in load factor as well as an increased proportion of the base-load mix operating at low capacity factors (Fig. 3) these 

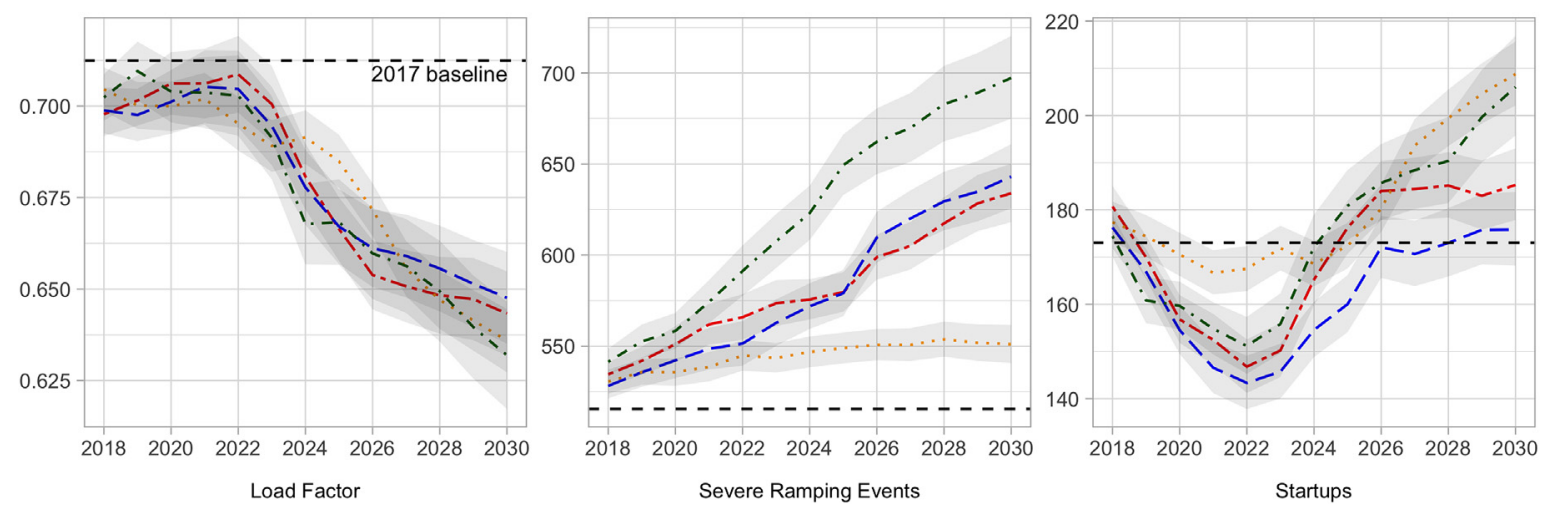

Scenario

- - Consumer Power

- - Slow Progress

Steady State

- . Two Degrees

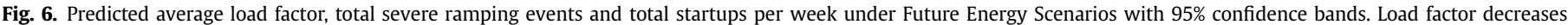

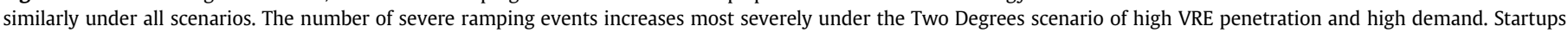

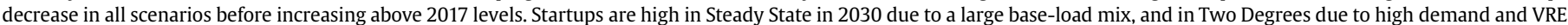
penetration.
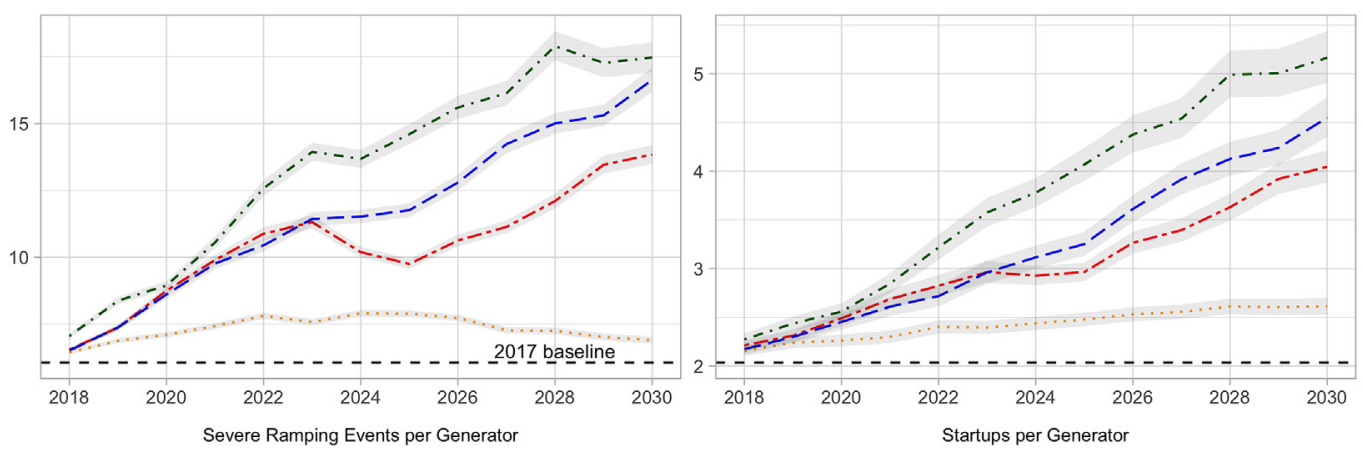

Scenario

--. Consumer Power

- - Slow Progress

Steady State

.. . Two Degrees

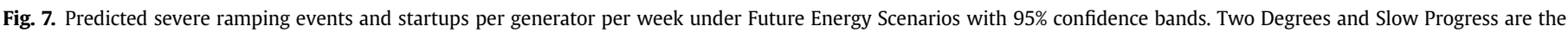

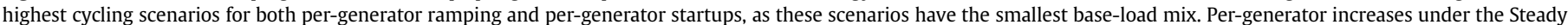
State scenario are comparatively slow, due to large predicted increases in CCGT capacity.

findings suggest that generating companies experienced increased operating costs during this period. At a start cost of $55 \$ 2012$ per MW capacity, ${ }^{3}$ the observed increase in per-generator startups between 2009 and 2017 represent an increase in operating costs of $8,300 £_{2012} 4$ per week for a typical 500 MW CCGT power station.

Inevitably, a significant proportion of the variance in the cycling variables could not be captured using the regression models. Fuel prices, carbon prices, scheduled and forced outages, demand and VRE forecast deviations and intra-day variations in demand are all likely to contribute to cycling. For short-term forecasting, these factors would be necessary for increasing model accuracy and capturing short-term variation. Our study focuses mainly on longterm scenario modelling and system-wide cycling at the annual resolution where variance is lower. Inclusion of variables such as those mentioned above would also limit applications of the models, as they are difficult to forecast several years into the future.

Using the regression models, we predicted reference-case cycling in the GB power system under Future Energy Scenarios. In comparison with the GB predictions for 2020 in [6], our predictions for the same year are much higher on a per generator level with increases of between $30-47 \%$ and $63-106 \%$ for startups and severe ramping events, respectively. VRE penetration in our Future Energy Scenarios are slightly higher, at $20-22 \%$ compared with $17-21 \%$ in

\footnotetext{
${ }^{3}$ Median warm CCGT start costs from [8].

4 Based on a yearly average spot rate of $\$ 1=£ 0.63$ for the year to 31 December 2012 [38].
}

[6]. Compared with simulation studies considering higher renewables penetrations, we generally predicted less severe cycling. In [10], the number of severe ramping events and startups per generator are predicted to increase by $500 \%$ and $150 \%$ respectively for penetrations of $20-40 \%$, compared with ranges of $14-190 \%$ and $28-150 \%$ respectively among scenarios in our study. Total startups are predicted to roughly double between 2020 and 2030 in Germany in [5], while we predict increases of between 3.6 and $21 \%$ between 2018 and 2030. For load factor, the decreases were similar to those predicted in simulation studies $[10,11]$.

The empirical, top-down approach adopted in this research does not allow us to account for the impact of technologies that may reduce cycling such as storage, demand-side response and interconnection, whose prevalence is projected to increase under the scenarios. As a result, our forecasts can only be seen as referencecase predictions for each scenario, with all other factors being equal. Nevertheless, the results do not apparently exhibit a bias towards strong cycling as compared with the simulation studies. This may be because our training data includes the period of large uptake open-cycle gas turbines after 2015 , which were observed to cycle heavily and mitigate base-load cycling.

The projected worsening of cycling would be significant for generating companies, representing further increases in long term O\&M costs and affecting the investment incentives for conventional generation [39]. The decrease in average load factor translates to reduced efficiency of base-load power stations that would impact their operating costs: for CCGTs, a reduction from $70 \%$ to $60 \%$ load factor results in an efficiency decrease of approximately 
$4 \%$ [9]. In addition, increased frequencies of startups and severe ramping events would result in higher operating costs for generating companies, although the extent of these increases differ considerably between scenarios. At a start cost of $55 \$_{2012}$ per MW capacity our predictions suggest that a typical 500 MW CCGT power station would incur increased operating costs of between 10,000 $£_{2012}$ (Steady State) and 54,000 $£_{2012}$ (Two Degrees) per week in 2030 compared with 2017 levels due to increased startups alone. Base-load generators are likely to rely increasingly on revenue opportunities from flexibility markets such as the Balancing Mechanism to supplement lost revenue from lower capacity factors and higher O\&M costs [39]. However, an important limitation of our research is that we do not capture the large variance in cycling among individuals in the generation mix. While some base-load generators are likely to experience very severe cycling behaviour (such as 'double two-shifting' regimes), others will not see such significant operational changes.

To the best of our knowledge, the models developed in this research are the first of their kind. As we demonstrated in Section 5.2 , they can be applied in scenario modelling to predict cycling rates without the need for high resolution dispatch models. They can be used as lightweight extensions to existing energy systems models to improve estimates for power station operating costs.

In addition, there are many applications of the open-access dataset which we do not address in this paper but we hope will be explored by the wider research community. For instance, a short-term forecasting model at the daily resolution could include more system variables such as fuel prices, carbon prices and intraday variations in demand and could be used to aid system operation and scheduling. We also see the dataset as having applications for benchmarking GB dispatch models.

\section{Conclusion}

This research aimed to investigate empirically the impact of VRE on the cycling of base-load generation in the GB power system. Using historical operational data, we developed a novel openaccess dataset on base-load cycling. Analysis of this data found that total startups remained roughly stable from 2009 to 2017, although startups per generator increased by $60 \%$. Load factor fell from $77 \%$ to $71 \%$, while severe ramping events fluctuated between 451 and 532 per week.

Our trained regression models demonstrated statistically significant links between VRE generation and the cycling variables. Using these models, we estimated that there would have been 35 fewer base-load startups per week in 2017, had VRE generation remained at 2009 levels. Likewise, load factor would not have decreased between 2009 and 2017, and severe ramping events would have been slightly lower.

The regression models can be applied in scenario modelling studies which was demonstrated through application to Future Energy Scenarios up to 2030. We found the number of startups and severe ramping events to increase by between $1.6-21 \%$ and $6.9-35 \%$ respectively, while load factor was projected to decrease from $71 \%$ to between 63 and 65\%. On a per generator level, the effects were more severe, with increases of up to $150 \%$ and $190 \%$ in the Two Degrees scenario for startups and severe ramping events respectively, due to fewer active base-load generators in the power system. For generating companies, this translates to considerable increases in operating costs during the 2020s.

\section{Acknowledgements}

This research was supported by an Engineering and Physical Sciences Research Council research studentship (grant number: EP/
$\mathrm{R} 512400 / 1)$.

\section{References}

[1] IRENA. Renewable energy statistics 2019. Tech. rep.. 2019.

[2] Strbac G, Shakoor A, Black M, Pudjianto D, Bopp T. Impact of wind generation on the operation and development of the UK electricity systems. Elec Power Syst Res 2007;77(9):1214-27. https://doi.org/10.1016/j.epsr.2006.08.014.

[3] Ringkjøb HK, Haugan PM, Solbrekke IM. A review of modelling tools for energy and electricity systems with large shares of variable renewables. Renew Sustain Energy Rev 2018;96(July):440-59. https://doi.org/10.1016 j.rser.2018.08.002.

[4] Eser P, Singh A, Chokani N, Abhari RS. Effect of increased renewables generation on operation of thermal power plants. Appl Energy 2016;164(2016): 723-32. https://doi.org/10.1016/j.apenergy.2015.12.017.

[5] Schill WP, Pahle M, Gambardella C. Start-up costs of thermal power plants in markets with increasing shares of variable renewable generation. Nat Energy 2017;2(6):1-6. https://doi.org/10.1038/nenergy.2017.50.

[6] Edmunds R, Davies L, Deane P, Pourkashanian M. Thermal power plant operating regimes in future British power systems with increasing variable renewable penetration. Energy Convers Manag 2015;105:977-85. https:// doi.org/10.1016/j.enconman.2015.08.067.

[7] Electric Power Research Institute (EPRI). Damage to power plants due to cycling. Tech. rep.. 2001.

[8] Kumar N, Besuner P, Lefton S, Agan D, Hilleman D. Power plant cycling costs. Tech. Rep. April. Sunnyvale, California: Intertek APTECH; 2012. https://doi.org/ $10.2172 / 1046269$.

[9] Van den Bergh K, Delarue E. Cycling of conventional power plants: technical limits and actual costs. Energy Convers Manag 2015;97:70-7. https://doi.org/ 10.1016/j.enconman.2015.03.026.

[10] Troy N, Denny E, O'Malley M. Base-load cycling on a system with significant wind penetration. IEEE Trans Power Syst 2010;25(2):1088-97. https:/ doi.org/10.1109/TPWRS.2009.2037326.

[11] Shortt A, Kiviluoma J, O'Malley M. Accommodating variability in generation planning. IEEE Trans Power Syst 2013;28(1):158-69. https://doi.org/10.1109/ TPWRS.2012.2202925.

[12] Lalor G, Member S, Mullane A, Malley MO, Member S. Frequency control and wind turbine technologies. IEEE Trans Power Syst 2005;20(4):1905-13. https://doi.org/10.1109/TPWRS.2005.857393.

[13] Bevrani H, Ghosh A, Ledwich G. Renewable energy sources and frequency regulation: survey and new perspectives. IET Renew Power Gener 2010;4(5): 438. https://doi.org/10.1049/iet-rpg.2009.0049.

[14] Sun YZ, Zhang ZS, Li GJ, Lin J. Review on frequency control of power systems with wind power penetration. In: 2010 International conference on power system technology: technological innovations making power grid smarter POWERCON2010; 2010. p. 1-8. https://doi.org/10.1109/ POWERCON.2010.5666151.

[15] Margaris ID, Papathanassiou SA, Hatziargyriou ND, Hansen AD, Sorensen P. Frequency control in autonomous power systems with high wind power penetration. IEEE Trans Sustain Energy 2012;3(2):189-99. https://doi.org/ 10.1109/TSTE.2011.2174660.

[16] Doherty R, O'Malley M. A new approach to quantify reserve demand in systems with significant installed wind capacity. IEEE Trans Power Syst 2005;20(2):587-95. https://doi.org/10.1109/TPWRS.2005.846206.

[17] Holttinen H, Milligan M, Ela E, Dobschinski J, Menemenlis N, Rawn B, Bessa RJ, Flynn D, Gomez-Lazaro E, Detlefsen NK. Methodologies to determine operating reserves due to increased wind power. IEEE Trans Sustain Energy 2012;3(4):713-23. https://doi.org/10.1109/tste.2012.2208207.

[18] De Vos K, Morbee J, Driesen J, Belmans R. Impact of wind power on sizing and allocation of reserve requirements. IET Renew Power Gener 2013;7(1):1-9. https://doi.org/10.1049/iet-rpg.2012.0085.

[19] Jabr RA. Robust transmission network expansion planning with uncertain renewable generation and loads. IEEE Trans Power Syst 2013;28(4):4558-67. https://doi.org/10.1109/TPWRS.2013.2267058.

[20] Brown T. Transmission network loading in Europe with high shares of renewables. IET Renew Power Gener 2014;9(1):57-65. https://doi.org/10.1049/ iet-rpg.2014.0114.

[21] Palsson M, Toftevaag T, Uhlen K, Tande J. Large-scale wind power integration and voltage stability limits in regional networks. In: IEEE power engineering society summer meeting, Chicago, IL, USA, 21-25 July 2002; 2002. p. 762-9. https://doi.org/10.1109/pess.2002.1043417.

[22] Vittal E, O'Malley M, Keane A. A steady-state voltage stability analysis of power systems with high penetrations of wind. IEEE Trans Power Syst 2010;25(1):433-42. https://doi.org/10.1109/TPWRS.2009.2031491.

[23] ESB National Grid. Impact of wind power generation in Ireland on the operation of conventional plant and the economic implications. Tech. rep.. 2004.

[24] Ummels BC, Gibescu M, Pelgrum E, Kling WL, Brand AJ. Impacts of wind power on thermal generation unit commitment and dispatch. IEEE Trans Energy Convers 2007;22(1):44-51. https://doi.org/10.1109/TEC.2006.889616.

[25] Meibom P, Weber C, Barth R, Brand H. Operational costs induced by fluctuating wind power production in Germany and Scandinavia. IET Renew Power Gener 2009;3(1). https://doi.org/10.1049/iet-rpg:20070075.

[26] Makarov YV, Loutan C, Ma J, de Mello P. Operational impacts of wind generation on California power systems. IEEE Trans Power Syst 2009;24(2): 
1039-50. https://doi.org/10.1109/TPWRS.2009.2016364. arXiv:0411295.

[27] Lew BD, Brinkman G. Finding flexibility: cycling the conventional fleet. IEEE Power Energy Mag 2013;11(6):20-32. https://doi.org/10.1109/ MPE.2013.2277988

[28] Huber M, Dimkova D, Hamacher T. Integration of wind and solar power in Europe: assessment of flexibility requirements. Energy 2014;69:236-46. https://doi.org/10.1016/j.energy.2014.02.109.

[29] Hermans M, Bruninx K, Delarue E. Impact of CCGT start-up flexibility and cycling costs toward renewables integration. IEEE Trans Sustain Energy 2018;9(3):1468-76. https://doi.org/10.1109/TSTE.2018.2791679.

[30] Mikkola J, Lund PD. Modeling flexibility and optimal use of existing power plants with large-scale variable renewable power schemes. Energy 2016;112: 364-75. https://doi.org/10.1016/j.energy.2016.06.082.

[31] Bass RJ, Malalasekera W, Willmot P, Versteeg HK. The impact of variable demand upon the performance of a combined cycle gas turbine (CCGT) power plant. Energy 2011;36(4):1956-65. https://doi.org/10.1016 j.energy.2010.09.020.

[32] Turconi R, Dwyer CO, Flynn D, Astrup T. Emissions from cycling of thermal power plants in electricity systems with high penetration of wind power : life cycle assessment for Ireland. Appl Energy 2014;131:1-8. https://doi.org/ 10.1016/j.apenergy.2014.06.006.

[33] Lefton SA, Besuner P. The cost of cycling coal fired power plants. In: Coal power magazine winter 2006; 2006. p. 16-20.

[34] Smith AZ, Halliday J. The P114 data set: disaggregate half-hourly demand and supply data on the GB electricity grid. Tech. rep. UKERC; 2016.

[35] National Grid. Demand data. 2018. URL, https://www.nationalgrideso.com/ balancing-data/data-finder-and-explorer.

[36] Elexon. BM reports. 2018. URL, https://www.bmreports.com.

[37] National Grid. Future energy scenarios. 2017. URL, http://fes.nationalgrid.com/ fes-document/fes-archives/.

[38] HMRC. Yearly average and spot rates. 2013. URL, https://www.gov.uk/ government/uploads/system/uploads/attachment_data/file/371053/Avgyear-20130331.csv/preview.

[39] Steggals W, Gross R, Heptonstall P. Winds of change: How high wind penetrations will affect investment incentives in the GB electricity sector. Energy Pol 2011;39(3):1389-96. https://doi.org/10.1016/j.enpol.2010.12.011. 